\title{
Impact of Data Retrieval Pattern on Homogeneous Signal Field Reconstruction in Dense Sensor Networks
}

\author{
Min Dong, Member, IEEE, Lang Tong, Fellow, IEEE, and Brian M. Sadler, Senior Member, IEEE
}

\begin{abstract}
We analyze the impact of data retrieval pattern on the reconstruction performance of a one-dimensional homogeneous random field measured by a large-scale sensor network. From a networking perspective, we connect data retrieval protocols and different sampling schemes. Specifically, we show that the data retrieval pattern affects the efficiency of reconstruction; as the number of received packets $M$ increases, the deterministic retrieval pattern that schedules sensors to transmit from equally spaced locations results in a faster decay of distortion than the random pattern does. In particular, we show that the ratio of the excess reconstruction distortion under the random retrieval pattern to that under the deterministic one grows as $\log M+O(\log \log M)$. Comparing the reconstruction performance directly, we further show that, in the high measurement signal-to-noise ratio (SNR) regime, the benefit from carefully scheduling sensor transmissions from specific locations instead of collecting in a random fashion is substantial. In the low SNR regime, however, using the random pattern results in little reconstruction performance loss. Finally, as $M \rightarrow \infty$, we show the strong convergence property of reconstruction distortion under the random pattern.
\end{abstract}

Index Terms-Data retrieval, estimation, random field, sampling, sensor network, signal reconstruction.

\section{INTRODUCTION}

\section{A. Motivation}

W E consider the problem of reconstructing a homogeneous random signal field using data collected from a sensor network. From a signal processing perspective, this is the classical problem of signal reconstruction from possibly random samples, and the literature is extensive. There is, however, a communication and networking aspect of the problem unique to sensor networks. That is how the data are collected from a sensor network, and whether one kind of data collection is better than another. Here we would be concerned about the simplicity of the collection protocol, whether it is energy

\footnotetext{
Manuscript received December 1, 2003; revised January 10, 2006. The associate editor coordinating the review of this manuscript and approving it for publication was Prof. Gregori Vazquez. This work was supported in part by the Multidisciplinary University Research Initiative under the Office of Naval Research Contract N00014-00-1-0564, the Army Research Laboratory CTA on Communication and Networks under Grant DAAD19-01-2-0011, and the National Science Foundation under Contract CCR-0435190.

M. Dong is with Corporate R\&D, Qualcomm Inc., San Diego, CA 92121 USA (e-mail: mdong@qualcomm.com).

L. Tong is with the School of Electrical and Computer Engineering, Cornell University, Ithaca, NY 14853 USA (e-mail: Itong @ece.cornell.edu).

B. M. Sadler is with the Army Research Laboratory, Adelphi, MD 20783

USA (e-mail: bsadler@arl.army.mil).

Digital Object Identifier 10.1109/TSP.2006.881233
}

efficient, and whether the reconstruction performance will be adequate for the specific application.

Take, for example, two different collection strategies. The first is to schedule transmissions from specific locations of the sensor field. A designer can carefully choose the sensor locations to collect data based on the properties of the signal ( e.g., temporal and spatial bandwidths, statistical correlations, etc.) so that the reconstruction error is minimized. While the reconstruction performance is optimized, the complexity of such a centralized scheduling approach can be prohibitive. Specifically, sensors have to be addressed individually, and the collection schedule must be disseminated. Furthermore, centralized scheduling may not be robust against sensor errors; if the batteries of some scheduled sensor run out, the optimization has to be performed again among sensors. The scheduling will be different, and it has to be redistributed.

An alternative is to consider a simple and distributed collection strategy. For example, the collection can be based on a random selection of sensors using simple protocols such as ALOHA and CSMA. Such a strategy can be implemented easily, especially for sensor networks with mobile access [1], [3]. Here a simple ALOHA strategy will result in the random sampling of the signal field. While the communication and networking protocol is simple, the reconstruction performance is affected.

For a network designer who, on the one hand, would like to reconstruct the signal field as accurately as possible and, on the other hand, must design the network that uses simple and energy efficient protocols, tradeoffs have to be made between performance and complexity. Such a tradeoff must be made with a cross-layer perspective, which connects application layer attributes such as the mean square error of the signal reconstruction with medium access control (MAC) layer functions.

\section{B. Contributions}

We present in this paper an analysis of the impact of data collection on the performance of signal reconstruction. We make the abstraction that different data collection (or MAC) protocols result in different sampling patterns, which in turn affect the reconstruction performance. While such an abstraction masks the details of physical and MAC layer implementations, it is valuable for the designers to gain insights.

We consider the reconstruction of a signal field using deterministic and random sampling schemes. By deterministic scheme we mean that samples from fixed sensor locations in the signal field are collected. For the random sampling scheme, in contrast, the origins of received samples are random in the sensor field with uniform distribution. One example of the 
MAC rendering such pattern is ALOHA, in which each sensor transmits with a certain probability. Therefore, a sample at one location is received with a certain probability. To make the problem more tractable, we study the performance in a one-dimensional (1-D) signal field, which provides insight into the two-dimensional problem.

Comparing the two sampling schemes analytically for a fixed number of received samples (packets) is difficult. Instead, we use asymptotic techniques by analyzing the scaling behavior of the reconstruction error with respect to the number of received samples $M$. Using the expected maximum reconstruction distortion as the performance metric, we obtain several asymptotic results.

We show first that the deterministic scheme that collects packets from equally spaced sensors is more efficient than the random scheme. This is not surprising. We provide, however, a quantitative characterization of the ratio of excess maximum distortions. In particular, the ratio of excess maximum distortion under the random sampling to that under the deterministic one grows with the number of collected packets $M$ as $\log M+O(\log \log M)$. In other words, the gain grows slowly with $M$. Next, we compare the reconstruction performance as a function of signal-to-noise ratio (SNR). We show that, in the high measurement SNR regime, the benefit from carefully scheduling transmission location instead of random sampling is substantial. At low SNR, however, using the random sampling results in little performance loss. Finally, we show the strong convergence of the random sampling scheme. That is, as $M \rightarrow \infty$, the maximum distortion in any realization converges to a deterministic value both with probability one and in mean square sense.

\section{Related Work and Organization}

While the mathematical problems considered in this paper are discussed in the context of sensor networks, they have close ties to the classical sampling problems. A key difference, however, is that we are more interested in the problem of data collection in a large but finite field. Thus the problem of signal reconstruction in a sensor network needs to be treated under the finite window setup, whereas in the classical sampling problem, the signal reconstruction window is usually infinite.

The sampling problem has been extensively studied in the literature. Many studies and performance analyses on the interpolation methods based on a periodic or random sample scheme have been conducted. See [4]-[6] and references therein. Interpolation, sequential estimation, or prediction using random sampling has also been studied [6]-[9]. The comparisons of periodic sampling with Poisson sampling, and time-jittering effect on periodic sampling, have been considered for some special cases [6], [10], [11].

Since the pattern of data collection is generated by the MAC protocol used in the data collection, our problem is indirectly related to the MAC design for sensor networks. Many MAC protocols have been proposed aiming to the special needs and requirements for both ad hoc sensor networks [12]-[15] and reachback sensor networks [2], [3]. Among these protocols, system throughput and energy efficiency are the major considerations. For applications such as estimating the signal field using a sensor network, how the underlying sampling pattern of these MAC schemes actually affects the estimation and reconstruction performance has not been addressed.

This paper is organized as follows. In Section II, we introduce models for the source and data collections followed by the descriptions of the estimator and distortion measure for signal reconstruction. In Section III, we obtain the formulas for the reconstruction distortion under both schemes for a given number of received data packets. We then provide the asymptotic performance comparison under the two retrieval schemes in Section IV. Numerical results are presented in Section V. We conclude in Section VI with comments on limitations of our result.

\section{Problem Statement}

\section{A. Source Model}

Consider a one-dimensional field of length $D$ denoted by $\mathcal{A}=[0, D]$. Let $S_{t}(x)(x \in \mathcal{A})$ be the source of interest in $\mathcal{A}$ at time $t$. We assume that the spatial dynamic of $S_{t}(x)$ is a one-dimensional homogeneous Gaussian random field ${ }^{1}$ governed by the following linear stochastic differential equation:

$$
d S_{t}(x)=-f S_{t}(x) d x+\sigma d W_{t}(x)
$$

where $f>0$ and $\sigma \in \mathbf{R}$ are known. The process $W_{t}(x)$ is a standard Brownian motion, and the source signal $S_{t}(x)$ is the stationary solution of (1) with $S_{t}(x) \sim \mathcal{N}\left(0, \sigma^{2} / 2 f\right)$. Furthermore, it can be shown that $S_{t}(x)$ is both Gaussian and Markovian (details can be found in [16]). Given $S_{t}\left(x_{0}\right)$, we have

$$
\begin{aligned}
E\left\{S_{t}(x) \mid S_{t}\left(x_{0}\right)\right\} & =e^{\left[\int_{x_{0}}^{x}-f d x\right]} S_{t}\left(x_{0}\right) \\
& =e^{-f\left(x-x_{0}\right)} S_{t}\left(x_{0}\right) \quad\left(x_{0}<x\right) .
\end{aligned}
$$

Being homogeneous in $\mathcal{A}, S_{t}(x)$ has the autocorrelation

$$
\begin{aligned}
E\left\{S_{t}\left(x_{0}\right) S_{t}\left(x_{1}\right)\right\} & =E\left\{S_{t}\left(x_{0}\right) E\left\{S_{t}\left(x_{1}\right) \mid S_{t}\left(x_{0}\right)\right\}\right\} \\
& =\frac{\sigma^{2}}{2 f} e^{-f\left(x_{1}-x_{0}\right)}, \quad\left(x_{0}<x_{1}\right)
\end{aligned}
$$

which is only a function of distance between the two points $x_{1}$ and $x_{0}$. The process $S_{t}(x)$ described in (1) is also called Ornstein Uhlenbeck process. It is often used to model many physical phenomenons such as temperature, vibration, etc.

At a preprogrammed time $t$, every sensor measures the signal field, and the measurement of a sensor at location $x$ is given by

$$
Y_{t}(x)=S_{t}(x)+N_{t}(x)
$$

where $N_{t}(x)$ is spatially independent and identically distributed (i.i.d.) zero-mean white Gaussian measurement noise with variance $\sigma_{N}^{2}$, and it is assumed to be independent of $S_{t}(x)$. Each sensor then stores its local signal measurement in the form of a packet. Without loss of generality, we assume one measurement at $t$ is contained in a packet. Also included in the packet

\footnotetext{
${ }^{1}$ It is in general also called stationary Gaussian stochastic process. Here, we emphasize the parameter of the process being location instead of time.
} 


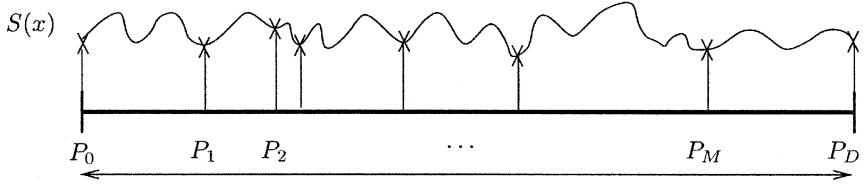

$D$

Fig. 1. A 1-D signal field sampled by a sensor network.

is the location information of the sensor obtained through some positioning method. Note that we reconstruct the source signal $\left\{S_{t}(x): x \in \mathcal{A}\right\}$ at time $t$ based only on the sensor measurements at time $t$. Therefore, we drop the time index for brevity in the following presentation.

\section{B. Two Types of Retrieval Schemes}

When the access point is ready for data collection, sensors transmit their packets to the access point. Each received data is a (noisy) sample of the signal field. Based on the resulting received sample location pattern in the field, we consider deterministic and random retrieval (sampling) schemes.

A deterministic scheme schedules transmissions according to a fixed sampling pattern. In other words, the access point draws $M$ samples from predetermined locations. One particular deterministic retrieval scheme is to schedule equally spaced sensors to access the channel. It turns out that this is the best deterministic scheme for the distortion measure defined below (see Section III), and we will refer to this as the deterministic scheme and denote it as $\pi_{u}$. Here we assume that sensors are densely deployed, and the deterministic scheme can always find sensors at desired locations.

For the random retrieval scheme, the origins of the $M$ received samples, denoted as $\left\{P_{1}, \ldots, P_{M}\right\}$, are random with uniform distribution. This appears as if the access point randomly samples the sensor measurement data in $\mathcal{A}$. We denote this scheme as $\pi_{r}$. Again, we assume that the sensor density is high enough that the access point can always receive a sufficiently large number of samples.

\section{Data Retrieval}

We assume that the access point receives a total of $M$ samples originated from some points in $\mathcal{A}$. To avoid the boundary effect for signal reconstruction, we assume that, during the data collection, the access point always obtains the samples from the two boundaries of $\mathcal{A}$. We denote the locations of this two edges by $P_{0}=0$ and $P_{D}=D$. The locations from which these $(M+2)$ packets are received are then denoted by $\mathbf{p}_{M}=$ $\left\{P_{0}, P_{1}, \ldots, P_{M}, P_{D}\right\}$. Fig. 1 shows an example of resulting sampling of signal field in $\mathcal{A}$. The signal field is then reconstructed based on the received data samples.

\section{Source Estimation Distortion}

Let the measurement signal-to-noise ratio be $\mathrm{SNR}=$ $\sigma^{2} / 2 f \sigma_{N}^{2}$. Denote the reconstructed signal by $\hat{S}(x)$. We define the maximum field reconstruction distortion by the maximum mean square estimation error in $\mathcal{A}$

$$
\mathcal{E}\left(\mathbf{p}_{M}, \mathrm{SNR}\right) \triangleq \max _{x \in \mathcal{A}} E\left\{|\hat{S}(x)-S(x)|^{2} \mid \mathbf{p}_{M}\right\}
$$

A retrieval scheme $\pi$ specifies how data packets should be transmitted. It, therefore, determines how the signal field $\{S(x): x \in \mathcal{A}\}$ is sampled. Consequently, $\pi$ specifies the distribution of sample points $\mathbf{p}_{M}$. Under a given retrieval scheme $\pi$, the expected maximum distortion of the signal field is then given by

$$
\overline{\mathcal{E}}(M, \mathrm{SNR} ; \pi) \triangleq E\left\{\mathcal{E}\left(\mathbf{p}_{M}, \mathrm{SNR}\right) \mid M, \pi\right\}
$$

where the expectation is taken over $\mathbf{p}_{M}$ for a given $M$.

We note that the reason to chose maximum mean squared error (MSE) rather than average MSE as the performance measure is that, in some sensor applications such as environmental monitoring, one may be more interested in areas that significant changes occur, and the use of average may conceal important change from detection. Thus it is justified to minimize the maximum distortion.

\section{E. The Choice of Estimator}

Given the $(M+2)$ received samples from location $\mathbf{p}_{M}=\left[P_{0}, P_{1}, \ldots, P_{M}, P_{D}\right]$, we denote the corresponding order statistics of the sample generation locations $\left\{P_{1}, \ldots, P_{M}\right\}$ by $P_{(1)}<\cdots<P_{(M)}$. We estimate the signal $S(x)$ at $x$ by MMSE smoothing based on all received data samples

$$
\hat{S}^{\mathrm{opt}}(x)=E\left[S(x) \mid\left\{Y(p), p \in \mathbf{p}_{M}\right\}\right], \quad x \in \mathcal{A} .
$$

Let $\mathbf{S}_{p_{M}}=\left[S\left(P_{0}\right), S\left(P_{(1)}\right), \ldots, S\left(P_{(M)}\right), S\left(P_{D}\right)\right]^{T}$ be the source signal of which the noisy measurements are received at the access point. The minimum MSE (MMSE) of $\hat{S}^{\text {opt }}(x)$ is given by

$$
\begin{aligned}
\mathcal{M}\left(x, \mathrm{SNR} ; \mathbf{p}_{M}\right) & \triangleq E\left\{\left|\hat{S}^{\mathrm{opt}}(x)-S(x)\right|^{2} \mid \mathbf{p}_{M}\right\} \\
& =\frac{\sigma^{2}}{2 f}-\mathbf{r}\left(x, \mathbf{p}_{M}\right)\left(\mathbf{R}_{\mathbf{p}_{M}}+\sigma_{N}^{2} \mathbf{I}\right)^{-1} \mathbf{r}^{H}\left(x, \mathbf{p}_{M}\right)
\end{aligned}
$$

where $\mathbf{r}\left(x, \mathbf{p}_{M}\right) \triangleq E\left\{S(x) \mathbf{S}_{p_{M}}^{H}\right\}$ and $\mathbf{R}_{\mathbf{p}_{M}} \triangleq E\left\{\mathbf{S}_{p_{M}} \mathbf{S}_{p_{M}}^{H}\right\}$. Then, we have

$$
\mathcal{E}\left(\mathbf{p}_{M}, \mathrm{SNR}\right)=\max _{y \in \mathcal{A}} \mathcal{M}\left(y, \mathrm{SNR} ; \mathbf{p}_{M}\right) .
$$

The optimal MMSE estimator is in general difficult to analyze. To find the maximum distortion, one needs to search among the MSEs at all locations in $\mathcal{A}$, which does not result in a simple and clear solution.

We consider from now on a suboptimal estimator that uses the two immediate neighbor samples. Specifically, the estimator is given by

$$
\hat{S}(x)=E\left[S(x) \mid\left\{Y\left(P_{(i)}\right), Y\left(P_{(i+1)}\right)\right\}\right], \quad P_{(i)}<x<P_{(i+1)} \cdot
$$

When there is no measurement noise, this simple estimator is indeed optimal thanks to the Markovian nature of the source.

Our goal next is to analyze the signal field reconstruction performance under the two types of retrieval schemes. Specifically, we analyze how the expected maximum distortion $\overline{\mathcal{E}}(M, \mathrm{SNR} ; \pi)$ varies with $M$ and $\pi$. For this goal, we adopt the suboptimal MMSE estimator in (9) for signal reconstruction which is optimal in the noiseless measurement case. By 
analyzing its performance under different schemes, we wish to shed some light on how the fundamentally different retrieval approaches and measurement SNR affect the reconstruction performance.

\section{Calculating Reconstruction Distortion}

In this paper, we consider the case when the sensor density goes to infinity. In other words, there exists a sensor at any desired locations of $\mathcal{A}$. As we have mentioned earlier, under the random sampling, the sample from each sensor has equal probability to be received. Therefore, given $M$ received samples 2 at the access point, their original locations $\left\{P_{1}, \ldots, P_{M}\right\}$ can be modeled as i.i.d. random variables with uniform distribution $U(0, D)$. In contrast, for a deterministic scheme, location points $\left\{P_{1}, \ldots, P_{M}\right\}$ are fixed. In particular, for $\pi_{u}$, we have $\mathbf{p}_{M}=\{0, D /(M+1), 2 D /(M+1), \ldots, D\}$.

\section{A. The Expected Maximum Distortion}

By the Gaussian property of the process $\{S(x)\}$, the MMSE estimator in (9) is obtained as

$$
\begin{aligned}
\hat{S}(x) & =E\left\{S(x) \mathbf{Y}_{p}^{(i)}{ }^{H}\right\}\left(E\left\{\mathbf{Y}_{p}^{(i)} \mathbf{Y}_{p}^{(i)}{ }^{H}\right\}\right)^{-1} \mathbf{Y}_{p}^{(i)} \\
& =E\left\{S(x) \mathbf{S}_{p}^{(i)}{ }^{H}\right\}\left(E\left\{\mathbf{S}_{p}^{(i)} \mathbf{S}_{p}^{(i)}{ }^{H}\right\}+\sigma_{n}^{2} \mathbf{I}\right)^{-1} \mathbf{Y}_{p}^{(i)}(10)
\end{aligned}
$$

where $P_{(i)}<x<P_{(i+1)}, \mathbf{Y}_{p}^{(i)}=\left[Y\left(P_{(i)}\right), Y\left(P_{(i+1)}\right)\right]^{T}$, and $\mathbf{S}_{p}^{(i)}=\left[S\left(P_{(i)}\right), S\left(P_{(i+1)}\right)\right]^{T}$. Using the correlation of $S(x)$ in (3), the MSE of $\hat{S}(x)$ can then be obtained by

$$
\begin{aligned}
E\left\{|\hat{S}(x)-S(x)|^{2} \mid P_{(i)}, P_{(i+1)}\right\} & \\
= & E|S(x)|^{2} \\
& -E\left\{S(x) \mathbf{S}_{p}^{(i)^{T}}\right\}\left(E\left\{\mathbf{S}_{p}^{(i)} \mathbf{S}_{p}^{(i)^{T}}\right\}+\sigma_{n}^{2} \mathbf{I}\right)^{-1} E\left\{\mathbf{S}_{p}^{(i)} S(x)\right\} \\
= & \frac{\sigma^{2}}{2 f}[1 \\
& \left.-\frac{\left(\frac{1}{\mathrm{SNR}}+1\right)\left(e^{-2 f\left(x-P_{(i)}\right)}+e^{-2 f\left(P_{(i+1)}-x\right)}\right)-2 e^{-2 f d_{i}^{(M)}}}{\left(\frac{1}{\mathrm{SNR}}+1\right)^{2}-e^{-2 f d_{i}^{(M)}}}\right]
\end{aligned}
$$

where

$$
d_{i}^{(M)} \triangleq P_{(i+1)}-P_{(i)}
$$

${ }^{2}$ The actual number of received packets is $M+2$. For convenience, we only count those samples not from the two boundaries of $A$. for $i=0, \ldots, M .^{3}$ The superscript of $d_{i}$ denotes the total number of received data samples.

Finding the maximum distortion $\mathcal{E}\left(\mathbf{p}_{M}, \mathrm{SNR}\right)$ in (5) can be then broken down to finding the maximum distortion of $S(x)$ in each interval $P_{(i)}<x<P_{(i+1)}$, for $i=0, \ldots, M$

$$
\begin{aligned}
& \mathcal{E}\left(\mathbf{p}_{M}, \mathrm{SNR}\right) \\
& \left.\quad=\max _{0 \leq i \leq M} \max _{P_{(i)}<x<P_{(i+1)}} E\left\{|\hat{S}(x)-S(x)|^{2} \mid P_{(i)}, P_{(i+1)}\right\}\right\} .
\end{aligned}
$$

From (11), the maximum MSE of $\hat{S}(x)$, for $P_{(i)}<x<P_{(i+1)}$, is obtained at the midpoint of $P_{(i)}$ and $P_{(i+1)}$. It is only a function of $d_{i}^{(M)}$ and is given by (14) at the bottom of the page. Since $f>0, \mathcal{E}\left(d_{i}^{(M)}, \mathrm{SNR}\right)$ in (14) is an increasing function of $d_{i}^{(M)}$. Therefore, the maximum distortion $\mathcal{E}\left(\mathbf{p}_{M}, \mathrm{SNR}\right)$ in (13) is determined by the maximum of distance between any two adjacent data samples

$$
\begin{aligned}
\mathcal{E}\left(\mathbf{p}_{M}, \mathrm{SNR}\right) & =\max _{0 \leq i \leq M} \mathcal{E}\left(d_{i}^{(M)}, \mathrm{SNR}\right) \\
& =\frac{\frac{1}{\mathrm{SNR}}+1-e^{-f d_{\max }^{(M)}}}{\frac{1}{\mathrm{SNR}}+1+e^{-f d_{\max }^{(M)}}} \frac{\sigma^{2}}{2 f} \\
& \triangleq \mathcal{E}\left(d_{\max }^{(M)}, \mathrm{SNR}\right)
\end{aligned}
$$

where

$$
d_{\max }^{(M)}=\max _{0 \leq i \leq M} d_{i}^{(M)} .
$$

The average maximum distortion in (6) is then given by

$$
\overline{\mathcal{E}}(M, \mathrm{SNR} ; \pi)=E\left\{\mathcal{E}\left(d_{\max }^{(M)}, \mathrm{SNR}\right) \mid M, \pi\right\}
$$

where the expectation is now taken over $d_{\max }^{(M)}$ for a given $M$ and $\pi$.

For a deterministic scheme, the locations from which the $M$ samples are drawn are predetermined, and therefore $d_{\max }^{(M)}$ is fixed. Notice that $d_{\max }^{(M)} \geq D /(M+1)$. It is clear that $\pi_{u}$ results in the minimum $d_{\max }^{(\bar{M})}(=D /(M+1))$, and thus the minimum distortion $\overline{\mathcal{E}}\left(M, \mathrm{SNR} ; \pi_{u}\right)$, among all deterministic retrieval schemes. Therefore

$$
\begin{aligned}
\overline{\mathcal{E}}\left(M, \mathrm{SNR} ; \pi_{u}\right) & =\mathcal{E}\left(\frac{D}{M+1}, \mathrm{SNR}\right) \\
& =\frac{\frac{1}{\mathrm{SNR}}+1-e^{-f(D /(M+1))}}{\frac{1}{\mathrm{SNR}}+1+e^{-f(D /(M+1))}} \frac{\sigma^{2}}{2 f} .
\end{aligned}
$$

In contrast, under the random sampling, $d_{\max }^{(M)}$ is random with a certain distribution. In this case, to calculate $\overline{\mathcal{E}}\left(M, \mathrm{SNR} ; \pi_{r}\right)$,

${ }^{3}$ We denote $P_{(0)}=0$ and $P_{(M+1)}=D$.

$$
\begin{aligned}
\mathcal{E}\left(d_{i}^{(M)}, \mathrm{SNR}\right) & \triangleq \max _{P_{(i)}<x<P_{(i+1)}} E\left\{|\hat{S}(x)-S(x)|^{2} \mid P_{(i)}, P_{(i+1)}\right\} \\
& =\left.\frac{\sigma^{2}}{2 f}\left[1-\frac{\left(\frac{1}{\mathrm{SNR}}+1\right)\left(e^{-2 f\left(x-P_{(i)}\right)}+e^{-2 f\left(P_{(i+1)}-x\right)}\right)-2 e^{-2 f d_{i}^{(M)}}}{\left(\frac{1}{\mathrm{SNR}}+1\right)^{2}-e^{-2 f d_{i}^{(M)}}}\right]\right|_{x=\left(P_{(i)}+P_{(i+1)}\right) / 2} \\
& =\frac{\frac{1}{\mathrm{SNR}}+1-e^{-f d_{i}^{(M)}} \frac{\sigma^{2}}{\frac{1}{\mathrm{SNR}}+1+e^{-f d_{i}^{(M)}}}}{2 f}
\end{aligned}
$$




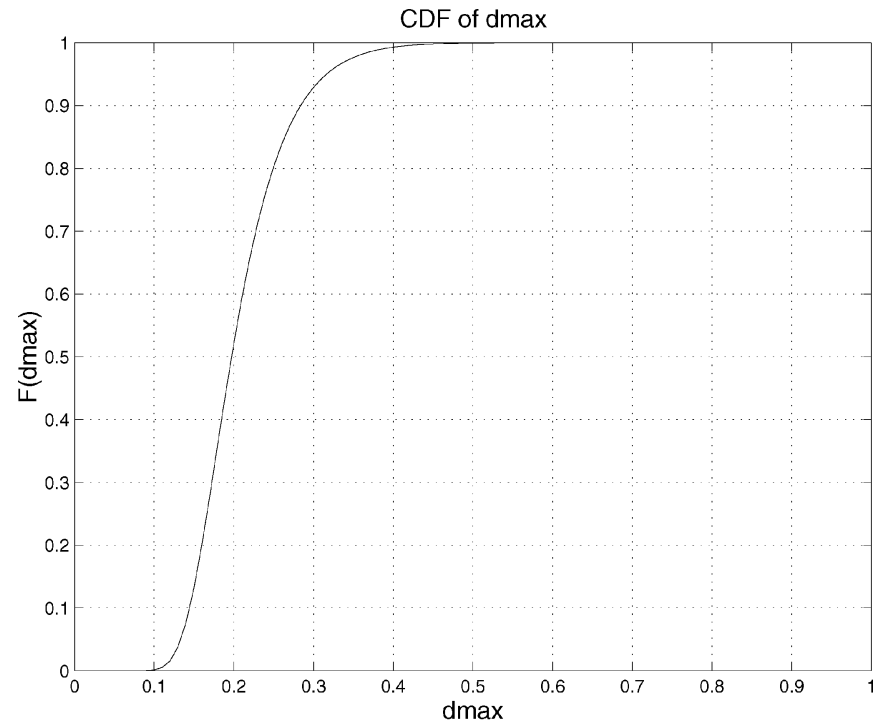

(a)

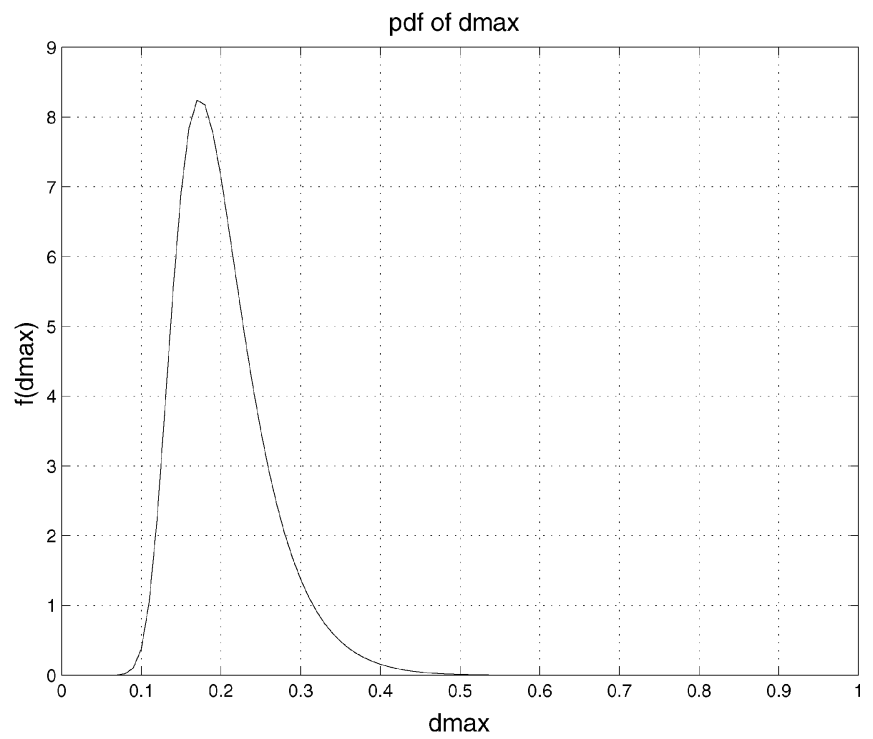

(b)

Fig. 2. The distribution of $d_{\max }^{(M)}$ under $\pi_{r}(M=15, D=1)$ : (a) cumulative distribution function of $d_{\max }^{(M)}$ and (b) probability density function of $d_{\max }^{(M)}$.

we need to find the probability distribution of the maximum sample distance $d_{\max }^{(M)}$, denoted by $F_{d^{(M)}}\left(\cdot ; \pi_{r}\right)$. It is given by the following proposition and is plotted in Fig. 2 for $M=15$ as an example.
Proposition 1: The probability distribution of the maximum sample distance $d_{\max }^{(M)}$ in the random retrieval scheme $\pi_{r}$ is given by (19) as shown at the bottom of the page.

Proof: See Appendix I.

Using (17) and Proposition $1, \overline{\mathcal{E}}\left(M, \mathrm{SNR} ; \pi_{r}\right)$ can be then

$$
\begin{aligned}
\overline{\mathcal{E}}_{\overline{\mathcal{E}}\left(M, \mathrm{SNR} ; \pi_{r}\right)} & =\int_{0}^{\infty} P\left(\mathcal{E}\left(d_{\max }^{(M)}, \mathrm{SNR}\right)>x ; \pi_{r}\right) d x \\
& =\int_{0}^{\mathcal{E}(D, \mathrm{SNR})} P\left(d_{\max }^{(M)}>t ; \pi_{r}\right) d \mathcal{E}(t, \mathrm{SNR}) \\
& =\int_{0}^{D}\left[1-F_{d_{\max }^{(M)}}\left(t ; \pi_{r}\right)\right] \mathcal{E}^{\prime}(t, \mathrm{SNR}) d t \\
& =\int_{0}^{D}\left[1-F_{d_{\max }^{(M)}}\left(t ; \pi_{r}\right)\right] \frac{2\left(\frac{1}{\mathrm{SNR}}+1\right) f e^{-f t}}{\left(\frac{1}{\mathrm{SNR}}+1+e^{-f t}\right)^{2}} d t
\end{aligned}
$$

where $t$ is such that $\mathcal{E}(t, \mathrm{SNR})=x$.

\section{B. The Distortion Decay Ratio}

We now compare the reconstruction performance under $\pi_{u}$ with that in the random retrieval scheme $\pi_{r}$. Let

$$
\overline{\mathcal{E}}_{\infty}(\mathrm{SNR} ; \pi) \triangleq \lim _{M \rightarrow \infty} \overline{\mathcal{E}}(M, \mathrm{SNR} ; \pi)
$$

be the limiting distortion under a specific SNR and $\pi$, as the number of received samples $M \rightarrow \infty$. For $\pi_{u}$, it is easy to see

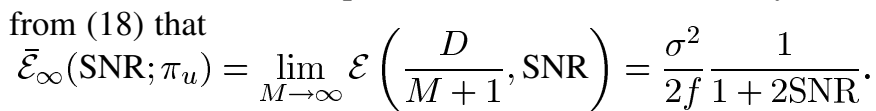

For $\pi_{r}$, we show in the proof of Theorem 1 in Section IV, (71), that $\overline{\mathcal{E}}_{\infty}\left(\mathrm{SNR} ; \pi_{r}\right)$ has the same expression as $\overline{\mathcal{E}}_{\infty}\left(\mathrm{SNR} ; \pi_{u}\right)$, i.e.,

$$
\overline{\mathcal{E}}_{\infty}\left(\mathrm{SNR} ; \pi_{r}\right)=\frac{\sigma^{2}}{2 f} \frac{1}{1+2 \mathrm{SNR}} .
$$

Having the same expression for the limiting distortion under $\pi_{u}$ and $\pi_{r}$ is expected. As the number of samples goes to infinity, we obtain the measurements of the whole field. The limiting distortion is therefore determined only by the accuracy of sensor measurements, i.e., the measurement SNR, not the specific scheme used.

The retrieval schemes, however, determine the value of the excess maximum distortion $\overline{\mathcal{E}}(M, \mathrm{SNR} ; \pi)-\overline{\mathcal{E}}_{\infty}(\mathrm{SNR} ; \pi)$. As $M$ increases, the number of received samples increases, and the

\footnotetext{
${ }^{4}$ We use the fact that, for $X \geq 0, E(X)=\int_{0}^{\infty} P(X>t) d t$.
}

$$
F_{d_{\text {max }}^{(M)}}\left(x ; \pi_{r}\right)= \begin{cases}0, & \text { if } 0 \leq x<\frac{D}{M+1} \\
\sum_{i=0}^{k}(-1)^{i}\left(\begin{array}{c}
M+1 \\
i
\end{array}\right)\left[(M-i+1) \frac{x}{D}-1\right]^{M}, & \text { if } \frac{D}{M-k+1} \leq x<\frac{D}{M-k}, \text { for } k=0, \ldots, M-2 \\
1-(M+1)\left(1-\frac{x}{D}\right)^{M}, & \text { if } \frac{D}{2} \leq x \leq D \\
1, & \text { if } x>D\end{cases}
$$


maximum distortion under both retrieval schemes decreases, but at different rates. Define the ratio of excess maximum distortion under $\pi_{r}$ to that under $\pi_{u}$ as

$$
r(M, \mathrm{SNR}) \triangleq \frac{\overline{\mathcal{E}}\left(M, \mathrm{SNR} ; \pi_{r}\right)-\overline{\mathcal{E}}_{\infty}\left(\mathrm{SNR} ; \pi_{r}\right)}{\overline{\mathcal{E}}\left(M, \mathrm{SNR} ; \pi_{u}\right)-\overline{\mathcal{E}}_{\infty}\left(\mathrm{SNR} ; \pi_{u}\right)} .
$$

Because $d_{\max }^{(M)}$ is the smallest under $\pi_{u}$, we have

$$
r(M, \mathrm{SNR}) \geq 1
$$

For any given number of received samples $M$, from (18) and (20), $r(M, \mathrm{SNR})$ can be calculated. As a function of $M$, $r(M, \mathrm{SNR})$ indicates the difference of the decreasing rate under $\pi_{r}$ and $\pi_{u}$.

\section{ASYMPTOTIC BEHAVIOR}

In this section, we address three issues relating to the asymptotic behavior of reconstruction distortion under the two sampling schemes. In Section IV-A, we consider the decay rate of the average maximum distortion under the two schemes when the number of received data samples $M$ is large but finite. This analysis reveals which scheme retrieves the data more efficiently. Specifically, by increasing $M$, the distortion under which the scheme reaches the limit, i.e., the limiting distortion, faster. In Section IV-B, we compare the actual distortions under the two sampling schemes, again, for finite but large $M$. This tells us the performance gap under the two schemes at a fixed SNR and $M$. In Section IV-C, we show the strong convergence of the random sampling scheme: as $M$ increases, the maximum distortion in any realization converges to a deterministic expression both with probability one and in mean square sense.

\section{A. Asymptotic Analysis of Decay Rate}

As mentioned earlier, when $M \rightarrow \infty$, we expect that the excess maximum distortion $\overline{\mathcal{E}}\left(M, \mathrm{SNR} ; \pi_{r}\right)-\overline{\mathcal{E}}_{\infty}(\mathrm{SNR} ; \pi)$ decreases to zero under both $\pi_{r}$ and $\pi_{u}$, but at different rates. We now compare the decay rates of distortion under the two sampling schemes by analyzing the asymptotic behavior of the ratio $r(M, \mathrm{SNR})$.

We show below that the asymptotic behavior of the ratio $r(M, \mathrm{SNR})$ is $\log M+O(\log \log M)$. We outline a few steps required to prove the result, leaving details to Appendix II. First, under the random sampling scheme $\pi_{r}$, we show that the probability distribution of the order statistics of the origins of $M$ received samples $\left\{P_{1}, \ldots, P_{M}\right\}$ is the same as the joint distribution of $M$ random variables, where each variable is a scaled summation of some i.i.d. exponential random variables. This allows us to link $d_{\max }^{(M)}$ to the maximum of i.i.d. exponential random variables. By the extreme value theory, as $M$ increases, the maximum of $M$ i.i.d. exponential random variables grows like $\log M$. Along with the strong law of large numbers, we show that $M d_{\max }^{(M)} / \log M$ converges to a constant with probability of one. Since the maximum distortion is determined by $d_{\max }^{(M)}$, it follows that the excess maximum distortion under $\pi_{r}$ scales like $\log M / M$. Since the excess maximum distortion under $\pi_{u}$ scales like $1 / M$, we then have the scaling behavior of the ratio $r(M, \mathrm{SNR})$.
Theorem 1: For reconstructing the one-dimensional homogeneous Gaussian random field described in (1), as $M \rightarrow \infty$, the ratio of the excess maximum reconstruction distortion under the random sampling scheme $\pi_{r}$ to that under the deterministic scheme $\pi_{u}$, defined as $r(M, \mathrm{SNR})$ in (23), is given by

$$
r(M, \mathrm{SNR})=\log M+O(\log \log M)+O\left(\frac{\log ^{2} M}{M}\right) .
$$

Proof: See Appendix II.

The intuition behind the growth rate of $r(M, \mathrm{SNR})$ being approximately $\log M$ is the following. The excess maximum distortion is determined only by the maximum distance $d_{\max }^{(M)}$ between any two adjacent sample locations (origins of received samples). Asymptotically, $d_{\max }^{(M)}$ under $\pi_{r}$ decreases as $\log M / M$, while under the uniform sampling $\pi_{u}$, it decreases as $1 / M$. Therefore, the distortion ratio $r(M, \mathrm{SNR})$ scales like $\log M$, as $M$ grows large.

Theorem 1 shows that the ratio of excess maximum distortion under $\pi_{r}$ to that under $\pi_{u}$ has a logarithmic growth rate. This in turn indicates that the retrieval pattern affects the decay rate of reconstruction distortion as the number of received samples increases, and therefore the efficiency of the reconstruction. Using the deterministic sampling $\pi_{u}$ results in the fastest decreasing rate of distortion over $M$. This scheme efficiency can also be interpreted in another way. The following corollary shows how many samples the access point needs to collect under each type of sampling scheme to reach the same reconstruction distortion level.

Corollary 1: Let $M_{\pi_{u}}$ and $M_{\pi_{r}}$ be the number of samples needed under $\pi_{u}$ and $\pi_{r}$ at the access point, respectively, to reconstruct the signal field with the same level of average maximum distortion. Then, as $\overline{\mathcal{E}}(M, \mathrm{SNR} ; \pi) \rightarrow \overline{\mathcal{E}}_{\infty}(\mathrm{SNR} ; \pi)$,

$$
M_{\pi_{u}}=O\left(\frac{M_{\pi_{r}}}{\log M_{\pi_{r}}}\right) \text {. }
$$

Proof: See Appendix III.

\section{B. Asymptotic Analysis of Distortion Ratio}

The previous comparison on the decay rate of distortion reveals the sampling efficiency on signal reconstruction under the deterministic and random sampling schemes. Another factor influences the reconstruction performance is the measurement SNR. As we will see, how sensitive the performance is to the variation of SNR is affected by the choice of retrieval pattern. This makes the performance gap under $\pi_{u}$ and $\pi_{r}$ appear to have different behaviors in different SNR regime. We now compare the actual average maximum distortion $\overline{\mathcal{E}}(M, \mathrm{SNR} ; \pi)$ under $\pi_{u}$ and $\pi_{r}$ directly in different SNR regimes. As mentioned earlier, the deterministic scheme $\pi_{u}$ is optimal in the sense that it results in the minimum signal reconstruction distortion. Define the ratio of average maximum distortion as

$$
\nu(M, \mathrm{SNR}) \triangleq \frac{\overline{\mathcal{E}}\left(M, \mathrm{SNR} ; \pi_{r}\right)}{\overline{\mathcal{E}}\left(M, \mathrm{SNR} ; \pi_{u}\right)}
$$

Then $\nu(M, \mathrm{SNR})$ (in $\mathrm{dB}$ ) indicates the performance gap between $\pi_{r}$ and $\pi_{u}$. We have the following result on the asymptotic behavior of $\nu(M, \mathrm{SNR})$. 
Theorem 2: As $M \rightarrow \infty$, the ratio of average maximum distortion $\nu(M, \mathrm{SNR})$ is given by

$$
\begin{array}{r}
\nu(M, \mathrm{SNR})=\frac{1}{1+\frac{C_{1}(\mathrm{SNR})}{M+1}\left(1+C_{1}(\mathrm{SNR}) \frac{\log M}{M}\right)} \\
+O\left(\frac{\log \log M}{M}\right)+O\left(\frac{\log ^{2} M}{M^{2}}\right)
\end{array}
$$

where

$$
C_{1}(\mathrm{SNR})=2 f D \mathrm{SNR} \frac{1+\mathrm{SNR}}{1+2 \mathrm{SNR}} .
$$

Proof: The proof is similar to that of Theorem 1 and is omitted.

1) High SNR Regime: For high SNR, the behavior of ratio $\nu(M, \mathrm{SNR})$ in Theorem 2 becomes the following.

Corollary 2: $\exists M_{0}$, such that for $\forall M>M_{0}, \exists \beta(M)$, such that for $\forall \mathrm{SNR}>\beta(M)$

$$
\nu(M, \mathrm{SNR}) \approx \log M+O\left(\frac{\log \log M}{M}\right)+O\left(\frac{\log ^{2} M}{M^{2}}\right) .
$$

Proof: See Appendix IV.

The above shows that for large $M$, when SNR is sufficiently high, the ratio $\nu(M, \mathrm{SNR})$ is $\log M$. In the extreme case, when SNR $\rightarrow \infty$, i.e., the measurements become noiseless, the limiting distortion $\overline{\mathcal{E}}_{\infty}(\mathrm{SNR} ; \pi)$ decreases to zero. In this case, from Theorem 1, as $M$ increases

$$
\nu(M, \infty)=r(M, \infty)=\log M+O(\log \log M)+O\left(\frac{\log ^{2} M}{M}\right) .
$$

This indicates that the reconstruction performance gap between $\pi_{r}$ and $\pi_{u}$ increases as $M$ increases. Thus, in the high SNR regime, as the number of received samples becomes large, the efficiency of the deterministic scheme $\pi_{u}$ translates to a large performance gain over the random sampling. Thus it may be justified to schedule carefully for a desired retrieval pattern instead of random sampling.

2) Low SNR Regime: In the low SNR regime, $\overline{\mathcal{E}}_{\infty}(\mathrm{SNR} ; \pi)$ is large. As $M$ increases, $\overline{\mathcal{E}}(M, \mathrm{SNR} ; \pi)$ decreases and is soon saturated to $\overline{\mathcal{E}}_{\infty}(\mathrm{SNR} ; \pi)$. Therefore, $\nu(M, \mathrm{SNR}) \rightarrow 1$. This can be also seen from Theorem 2. For low SNR, as $M$ becomes large, $\nu(M, \mathrm{SNR})$ in (27) becomes

$$
\nu(M, \mathrm{SNR}) \approx 1+O\left(\frac{\log M}{M}\right) .
$$

Comparing with the performance gain at high SNR, this indicates that the performance under $\pi_{u}$ is more sensitive to the measurement noise than that under $\pi_{r}$. For the latter, when the measurement SNR is low, despite the lower efficiency of the random sampling, it results in very little reconstruction performance loss, compared with $\pi_{u}$. In this regime, the reconstruction performance is dominated by the measurement noise and is less affected by the choice of different sampling schemes. The implementation simplicity of random sampling over scheduling sensor transmissions to form a desired pattern makes the former one more favorable.

\section{Strong Convergence of the Random Sampling}

Unlike the deterministic sampling $\pi_{u}$, where the maximum distortion for a given $M$ is fixed, under the random sampling $\pi_{r}$, since $d_{\text {max }}^{(M)}$ is random, the maximum distortion $\mathcal{E}\left(d_{\text {max }}^{(M)}\right.$, SNR $)$ in each realization is random. One can only evaluate the performance statistically. Our previous comparison of the two sampling schemes is based on the average performance evaluation. However, as $M$ becomes large, we show that the maximum distortion $\mathcal{E}\left(d_{\max }^{(M)}, \mathrm{SNR}\right)$ under $\pi_{r}$ converges to its mean, which has a deterministic expression. Therefore, for the distortion measure we consider, the random sampling can be viewed asymptotically as a deterministic sampling.

Theorem 3: As $M$ increases, the maximum distortion $\mathcal{E}\left(d_{\text {max }}^{(M)}\right)$ under $\pi_{r}$ converges to its mean $\overline{\mathcal{E}}\left(M, S N R ; \pi_{r}\right)$ with probability one. See (32) as shown at the bottom of the page, where for all large $M$

$$
\begin{aligned}
\overline{\mathcal{E}} & \left(M, S N R ; \pi_{r}\right) \\
& =\frac{\sigma^{2}}{2 f} \frac{\frac{1}{\mathrm{SNR}}+1-e^{-f(D(\log M / M)+O((\log \log M) / M))}}{\frac{1}{\mathrm{SNR}}+1+e^{-f(D(\log M / M)+O((\log \log M) / M))}} .
\end{aligned}
$$

Proof: From the expression in $(16), \mathcal{E}\left(d_{\max }^{(M)}, \mathrm{SNR}\right)$ is a continuous function of $d_{\mathrm{max}}^{(M)}$. Under $\pi_{r}$, from the almost sure convergence property of $d_{\max }^{(M)}$ in (68) of the proof of Theorem 1 , the result in (32) immediately follows.

Furthermore, since the maximum distortion is upper bounded

$$
\mathcal{E}\left(d_{\text {max }}^{(M)}, \mathrm{SNR}\right) \leq \mathcal{E}(D, \mathrm{SNR})
$$

following Theorem 3, we have the mean square convergence of $\mathcal{E}\left(d_{\text {max }}^{(M)}, \mathrm{SNR}\right)$ in the following corollary.

Corollary 3: As $M$ increases, the maximum distortion $\mathcal{E}\left(d_{\text {max }}^{(M)}, \mathrm{SNR}\right)$ under $\pi_{r}$ converges to $\overline{\mathcal{E}}\left(M, \mathrm{SNR} ; \pi_{r}\right)$ in mean square sense, i.e., the variance

$$
E\left|\mathcal{E}\left(d_{\max }^{(M)}, \mathrm{SNR} ; \pi_{r}\right)-\overline{\mathcal{E}}\left(M, \mathrm{SNR} ; \pi_{r}\right)\right|^{2} \rightarrow 0 \quad \text { as } M \rightarrow \infty .
$$

Proof: Since $\mathcal{E}\left(d_{\max }^{(M)}, \mathrm{SNR}\right)$ is bounded, by Theorem 3 and Scheffe's theorem [20], 5 the result immediately follows. Therefore, although our previous comparison of the sampling

$$
\text { 5If } X_{n} \stackrel{P}{\rightarrow} X \text { and }\left|X_{n}\right| \leq g \in L^{p} \text {, then } E\left|X_{n}-X\right|^{p} \rightarrow 0 .
$$

$$
\operatorname{Pr}\left(\forall \epsilon, \exists M_{0} \text {, s.t. } \forall M>M_{0},\left|\mathcal{E}\left(d_{\text {max }}^{(M)}, \mathrm{SNR}\right)-\overline{\mathcal{E}}\left(M, \mathrm{SNR} ; \pi_{r}\right)\right|<\epsilon\right)=1
$$




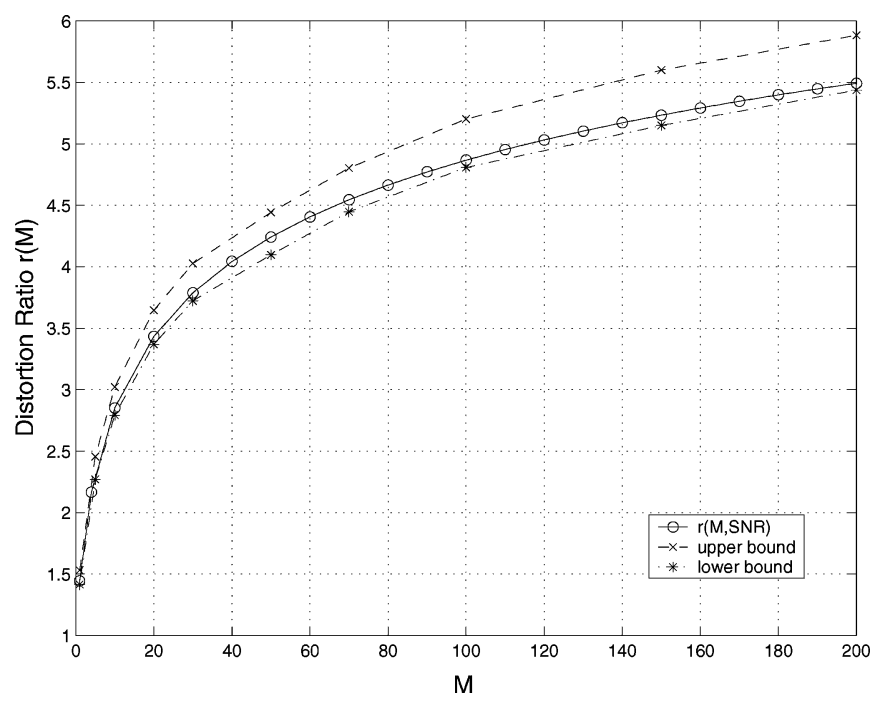

Fig. 3. $r(M, \mathrm{SNR})$ versus $M$ (noiseless). $f=0.2, \sigma^{2}=1, D=5$.

efficiency $r(M, \mathrm{SNR})$ is based on the average maximum distortion $\overline{\mathcal{E}}(M, \mathrm{SNR} ; \pi)$, the ratio of excess maximum distortion for any instance of realization of $M$ received data samples, i.e.,

$$
\frac{\mathcal{E}\left(d_{\text {max }}^{(M)}, \mathrm{SNR}\right)-\overline{\mathcal{E}}_{\infty}\left(\mathrm{SNR} ; \pi_{r}\right)}{\mathcal{E}\left(\frac{D}{M+1}, \mathrm{SNR}\right)-\overline{\mathcal{E}}_{\infty}\left(\mathrm{SNR} ; \pi_{u}\right)}
$$

converges to $r(M, \mathrm{SNR})$ with probability of one and in mean square sense. The same applies to the distortion ratio $\nu(M, \mathrm{SNR})$.

\section{NUMERICAL RESULTS}

\section{A. The Distortion Decay Rate}

We compute the ratio of excess maximum distortion $r(M, \mathrm{SNR})$ under various $M$. The ratio $r(M, \mathrm{SNR})$ is calculated based on (18), (20), and (23). To ease the computation, we can also use bounds on $r(M, \mathrm{SNR})$. Since $\mathcal{E}(x, \mathrm{SNR})$ is a concave function, the excess maximum distortion under $\pi_{r}$ can be bounded by the following inequality:

$\mathcal{E}_{l}(x, \mathrm{SNR}) \leq \mathcal{E}(x, \mathrm{SNR})-\overline{\mathcal{E}}_{\infty}\left(\mathrm{SNR} ; \pi_{r}\right) \leq \mathcal{E}_{u}(x, \mathrm{SNR})$,

$$
\text { for } x \in \mathcal{A}=[0, D]
$$

where

$$
\begin{aligned}
\mathcal{E}_{l}(x, \mathrm{SNR}) & =\frac{\mathcal{E}(D, \mathrm{SNR})-\mathcal{E}(0, \mathrm{SNR})}{D} x \\
& =\left(\frac{\frac{1}{\mathrm{SNR}}+1-e^{-f D}}{\frac{1}{\mathrm{SNR}}+1+e^{-f D}}-\frac{1}{1+2 \mathrm{SNR}}\right) \frac{x \sigma^{2}}{2 f D} \\
\mathcal{E}_{u}(x, \mathrm{SNR}) & =\mathcal{E}^{\prime}(0, \mathrm{SNR}) x=x \sigma^{2} \frac{\frac{1}{\mathrm{SNR}}+1}{\left(\frac{1}{\mathrm{SNR}}+2\right)^{2}} .
\end{aligned}
$$

The upper and lower bounds on the ratio of the maximum distortion are then computed using (18), (19) in Proposition 1, and (35).

Fig. 3 plots the ratio $r(M, \mathrm{SNR})$ and its upper and lower bounds versus $M$ for a small range of $M$ in the noiseless measurement case. We set $f=0.2, \sigma^{2}=1$, and $D=5$. We see

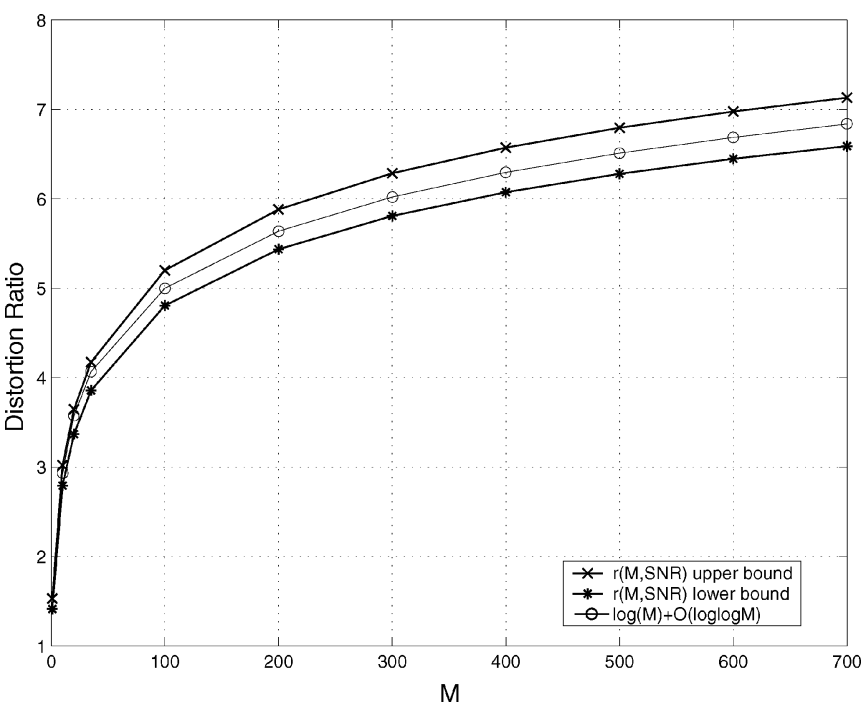

Fig. 4. Bounds of $r(M, \mathrm{SNR})$ versus $M$ (noiseless). $f=0.2, \sigma^{2}=1$, $D=5$.

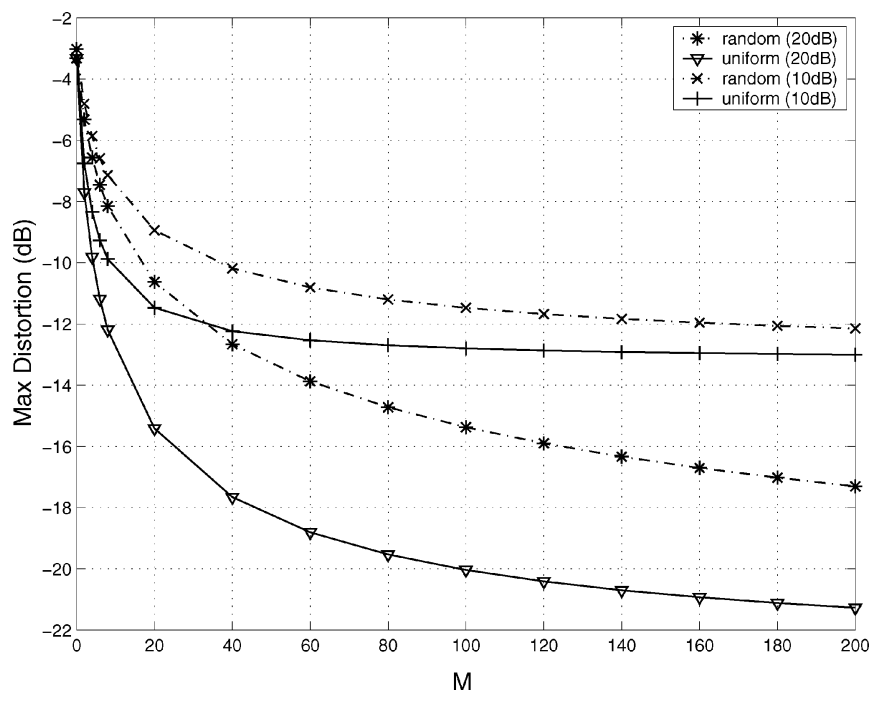

Fig. 5. Distortion ratio versus $M . f=0.2, \sigma^{2}=1, D=5$.

that the growth behavior of $r(M, \mathrm{SNR})$ can be well approximated by that of the two bounds. For a large range of $M$, we plot the upper and lower bounds of $r(M, \mathrm{SNR})$ in Fig. 4. Besides the bounds, we also plot the logarithm function $\log M+$ $O(\log \log M)$ as a comparison. We observe that, with $M$ greater than 200 , the growth rate of $r(M, \mathrm{SNR})$ is already approximately $\log M$, matching the asymptotic behavior of $r(M, \mathrm{SNR})$ in Theorem 1.

\section{B. The Expected Maximum Distortion $\overline{\mathcal{E}}(M, S N R ; \pi)$}

Fig. 5 shows the distortion curves $\overline{\mathcal{E}}(M, \mathrm{SNR} ; \pi)$ versus $M$ at SNR $=10$ and $20 \mathrm{~dB}$, respectively. They are computed based on (18) and (20). We see that the decay of the distortion performance under $\pi_{u}$ is faster than that under $\pi_{r}$. On the other hand, for a given target distortion tolerance level, we see that the required number of received samples under $\pi_{u}$ is much less than that under $\pi_{r}$. However, the performance of $\pi_{u}$ is sensitive to the noise level; it deteriorates faster. The performance gap 


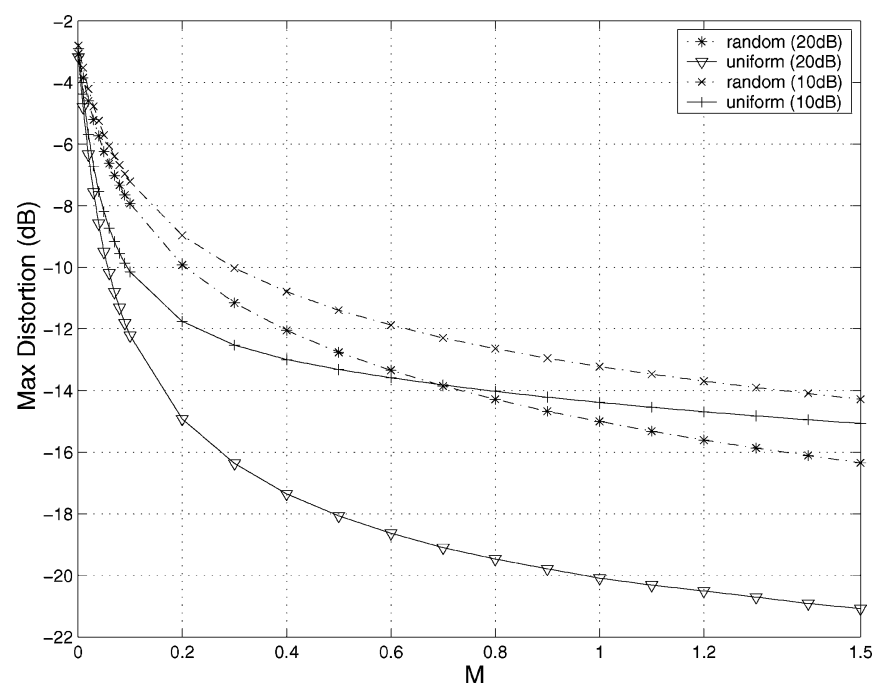

Fig. 6. Distortion versus $M$ using the optimal MMSE estimator in (7).

under the two different sampling schemes becomes smaller as the measurement noise become higher.

We have investigated the noise effect on the estimation performance using the simple suboptimal estimator. To see how the optimal estimator in (7) performs in the noisy case, we numerically calculate the average maximum distortion $\overline{\mathcal{E}}(M, \mathrm{SNR} ; \pi)$ using (8) and compare it under $\pi_{r}$ and $\pi_{u}$.

Fig. 6 shows the distortion curve versus $M$ at SNR = 10 and $20 \mathrm{~dB}$, respectively. The same setup as in Fig. 3 is used. Notice that, similar as in the suboptimal estimation case, the distortion performance under $\pi_{u}$ is sensitive to the noise level. The performance gap under the two different sampling schemes becomes smaller as the measurement noise become higher. This matches the intuition that as the measurement noise level becomes high, the performance is dominated by noise, and is less affected by the choice of different sampling schemes. Therefore, although our previous analysis is based on the suboptimal estimation, we expect that the similar performance behavior is preserved when the optimal estimator is used.

\section{CONCLUSION}

In sensor networks, due to the data correlations among sensor nodes, the choice of data collection scheme or medium access protocol and the resulting sampling pattern may significantly affect the performance of signal reconstruction. For reconstructing a one-dimensional dense signal field, we have shown that the deterministic sampling $\pi_{u}$ provides higher efficiency on reconstruction, i.e., it results in a faster decay rate of distortion than the random sampling $\pi_{r}$ does. In particular, the ratio of excess maximum distortion under $\pi_{r}$ to that under $\pi_{u}$ grows as $\log M+O(\log \log M)$. For high measurement $\mathrm{SNR}$, this translates to a meaningful performance gain of $\pi_{u}$ over $\pi_{r}$. We have also shown, however, that when the SNR is low, the difference on the performance between $\pi_{u}$ and $\pi_{r}$ becomes small; random sampling results in little reconstruction performance loss, and therefore, may be used instead of careful scheduling due to its simplicity. Finally, the convergence of the maximum distortion under $\pi_{r}$ to its deterministic mean demonstrates that the previous two asymptotic results apply to the comparison of the two sampling patterns for any instance of realization as well.

The analysis presented in this paper has a number of limitations. We have assumed a specific Gauss-Markov spatial process. Such a model is reasonable for diffusion processes but may not be appropriate for other types of signal field. The asymptotic results in this paper are obtained when sensor density goes to infinity. This ensures that polling $M$ data packets from desired sensor locations is possible under $\pi_{u}$, and we assume this can be done perfectly. This ideal assumption might not hold in practice due to imperfect transmission, reception, or dead sensors. Furthermore, for finite sensor density, there will exist partitions empty of sensors. All these situations result in missing data samples. As a consequence, the performance under the deterministic retrieval scheme may suffer from missing samples, and random retrieval schemes may result in better performance. To compare these two retrieval schemes in such situations, the approach will be different. How the reconstruction is affected in this case depends on the outage probability of sensors in a given region, and is under our investigation [21].

\section{APPENDIX I \\ PROOF OF PROPOSITION 1}

Proof: Under the random sampling schemes, given $M$ received data samples, we now find the cumulative distribution function $F_{d_{\max }^{(M)}}\left(\cdot ; \pi_{r}\right)$ of the maximum sample distance. For $\left\{P_{1}, \ldots, P_{M}\right\} \stackrel{i i d}{\sim} U(0, D),\left\{P_{(1)}, \ldots, P_{(M)}\right\}$ have the joint density given by [17]

$$
\begin{aligned}
f_{P_{(1)}, \ldots, P_{(M)}}\left(x_{1}, \ldots, x_{M}\right) \\
\quad= \begin{cases}\frac{M !}{D^{M}}, & \text { if } 0<x_{1}<\cdots<x_{M}<D \\
0, & \text { otherwise. }\end{cases}
\end{aligned}
$$

By the transformation of random variables, from (12) and (38), we have the joint distribution of sample distances $\left\{d_{0}, \ldots, d_{M-1}\right\}$ given by

$$
\begin{aligned}
& f_{d_{0}, \ldots, d_{M-1}}\left(x_{0}, \ldots, x_{M-1}\right) \\
& \quad= \begin{cases}\frac{M !}{D^{M}}, & \text { if } x_{i} \geq 0, \text { and } \sum_{i=0}^{M-1} x_{i} \leq D \\
0, & \text { otherwise. }\end{cases}
\end{aligned}
$$

Since $d_{M}=D-\sum_{i=0}^{M-1} d_{i}$, we have

$$
\begin{aligned}
F_{d_{\text {max }}^{(M)}}\left(x ; \pi_{r}\right) & =\operatorname{Pr}\left(d_{0} \leq x, \ldots, d_{M-1} \leq x, D-\sum_{i=0}^{M-1} d_{i} \leq x\right) \\
& =\int_{0}^{x} \cdots \int_{0}^{x} g\left(x_{0}, \ldots, x_{M-1}\right) d x_{0} \cdots d x_{M-1}(40)
\end{aligned}
$$

where

$$
\begin{gathered}
g\left(x_{0}, \ldots, x_{M-1}\right)=f_{d_{0}, \ldots, d_{M-1}}\left(x_{0}, \ldots, x_{M-1}\right) \\
\left.\cdot{ }^{\mathbf{1}}\left[D-\sum_{i=0}^{M-1} x_{i} \leq x\right]\right]^{\left(x_{0}, \ldots, x_{M-1}\right)}
\end{gathered}
$$

and $\mathbf{1}_{B}(x)$ is the indicator function

$$
\mathbf{1}_{B}(x)= \begin{cases}1, & \text { if } x \in B \\ 0, & \text { if } x \notin B\end{cases}
$$




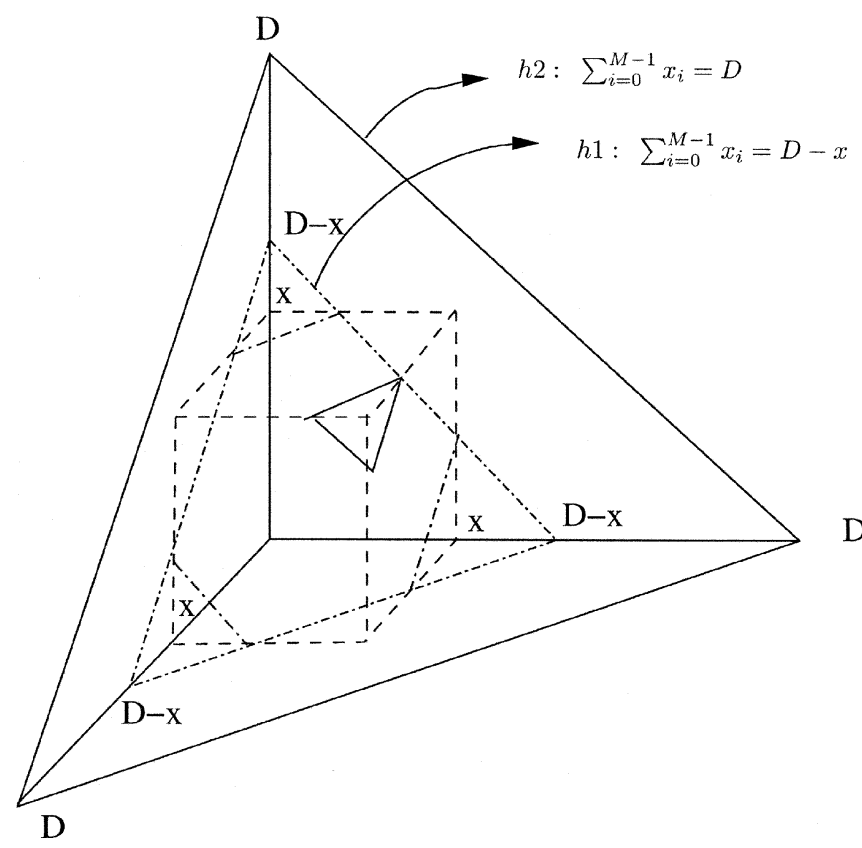

Fig. 7. Geometric representation of the integration in (40).

From (39)-(41), the integrations in (40) can essentially be viewed as the calculation of the volume of an $M$-dimensional ( $M$-dim) hypercube cut by two hyperplanes

$$
h_{1}: \sum_{i=0}^{M-1} x_{i}=D-x, \quad h_{2}: \sum_{i=0}^{M-1} x_{i}=D
$$

The equivalent volume to (40) is the part between $h_{1}$ and $h_{2}$. Denote this volume as $\mathcal{V}_{M}(x)$, then

$$
F_{d_{\max }^{(M)}}\left(x ; \pi_{r}\right)=\frac{M !}{D^{M}} \mathcal{V}_{M}(x)
$$

Fig. 7 shows the geometric interpretation for the three-dimensional case, where the cube has the volume of $x^{3}$. Denote the volume of the part of hypercube below the hyperplane $h_{1}$ and $h_{2}$ as $\mathcal{V}_{h_{1}}(x)$ and $\mathcal{V}_{h_{2}}(x)$, respectively. Then

$$
\mathcal{V}_{M}(x)=\mathcal{V}_{h_{2}}(x)-\mathcal{V}_{h_{1}}(x)
$$

The calculation of the volume of the cube between $h_{1}$ and $h_{2}$ is divided into the following cases.

1) For $0 \leq x<D /(M+1)$ : From geometry we know that, when $x \leq D / M$, the hypercube is not cut by $h_{2}$. In this case, $x \leq D /(M+1) \leq(D-x) / M$. The hypercube is under both $h_{1}$ and $h_{2}$. Therefore

$$
F_{d_{\max }^{(M)}}\left(x ; \pi_{r}\right)=\mathcal{V}_{M}=0 .
$$

2) For $D / 2 \leq x \leq D$ : In this case, the portion of the hypercube below $h_{1}$ is a regular $M$-dimensional hyperpyramid defined by

$$
\begin{aligned}
\mathcal{V}_{p_{M}}(a) & \triangleq \int_{0}^{a} \int_{0}^{a-x_{1}} \cdots \int_{0}^{a-x_{M-1}} d x_{M} \cdots d x_{1} \\
& =\frac{a^{M}}{M !}
\end{aligned}
$$

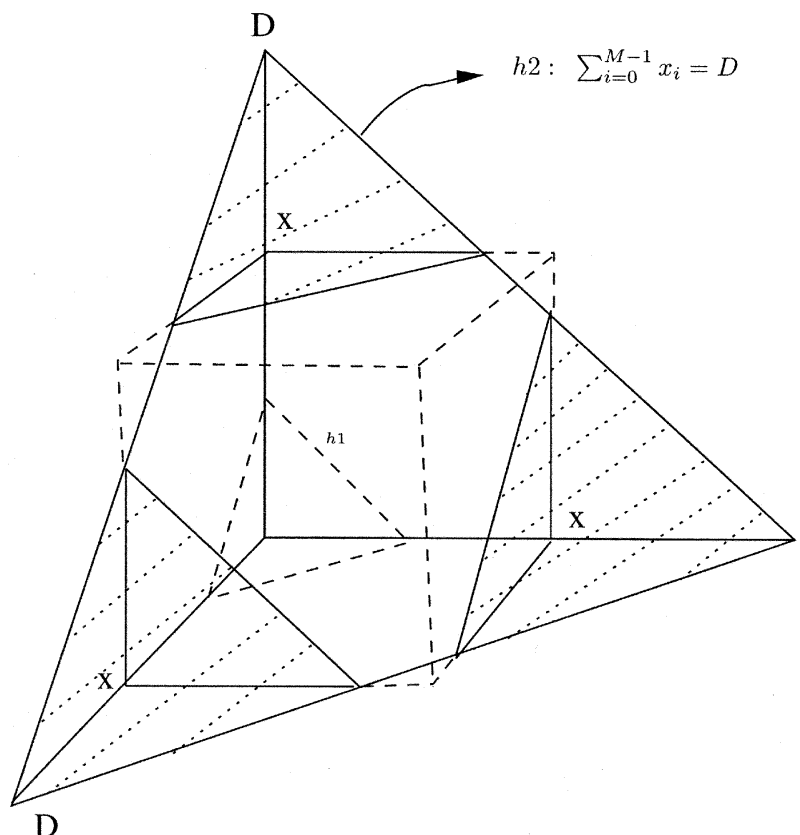

Fig. 8. A three-dimensional example in case 2).

Therefore

$$
\mathcal{V}_{h_{1}}(x)=\mathcal{V}_{p_{M}}(D-x)=\frac{(D-x)^{M}}{M !}
$$

The volume of the hypercube below by $h_{2}$ is simply the regular $M$-dimensional hyperpyramid with each of its "edges" another small $M$-dimensional hyperpyramid, cut off. An example in the three-dimensional case is shown in Fig. 8, where the shaded volumes are the "edges." Therefore, this volume can be obtained by

$$
\mathcal{V}_{h_{2}}(x)=\frac{D^{M}}{M !}-M \frac{(D-x)^{M}}{M !} .
$$

Finally, $\mathcal{V}_{M}(x)$ is given by

$\mathcal{V}_{M}(x)=\mathcal{V}_{h_{2}}(x)-\mathcal{V}_{h_{1}}(x)=\frac{D^{M}}{M !}\left(1-(M+1)\left(1-\frac{x}{D}\right)^{M}\right)$

Thus, $F_{d_{\max }}\left(x \mid M ; \pi_{r}\right)=1-(M+1)(1-x / D)^{M}$.

3) For $D /(M-k+1) \leq x<D /(M-k), k=$ $0, \ldots, M-2$ :

When $k=0$, the hypercube is not cut by $h_{2}$. Therefore

$$
\mathcal{V}_{h_{2}}(x)=x^{M}, \quad k=0 \text {. }
$$

For $k=1, \ldots, M-2, \mathcal{V}_{h_{2}}(x)$ is given by

$\mathcal{V}_{h_{2}}(x)=x^{M}-\sum_{i=0}^{k-1}(-1)^{i}\left(\begin{array}{c}M \\ i\end{array}\right) \frac{((M-i) x-D)^{M}}{M !}$
$k=1, \ldots, M-2$.

Similarly, $\mathcal{V}_{h_{1}}(x)$ is given by

$\mathcal{V}_{h_{1}}(x)=x^{M}-\sum_{i=0}^{k}(-1)^{i}\left(\begin{array}{c}M \\ i\end{array}\right) \frac{((M-i) x-(D-x))^{M}}{M !}$. 
Therefore

$$
\begin{aligned}
\mathcal{V}_{M}(x)= & \mathcal{V}_{h_{2}}(x)-\mathcal{V}_{h_{1}}(x) \\
= & \sum_{i=0}^{k}(-1)^{i}\left(\left(\begin{array}{c}
M \\
i
\end{array}\right) \frac{((M-i+1) x-D)^{M}}{M !}\right. \\
& -\sum_{i^{\prime}=1}^{k}(-1)^{(i \prime-1)}\left(\begin{array}{c}
M \\
i^{\prime}-1
\end{array}\right) \frac{\left(\left(M-i^{\prime}+1\right) x-D\right)^{M}}{M !} \\
= & \sum_{i=1}^{k}(-1)^{i}\left(\left(\begin{array}{c}
M \\
i
\end{array}\right)+\left(\begin{array}{c}
M \\
i-1
\end{array}\right)\right) \frac{((M-i+1) x-D)^{M}}{M !} \\
& +\frac{((M+1) x-D)^{M}}{M !} \\
= & \sum_{i=0}^{k}(-1)^{i}\left(\begin{array}{c}
M+1 \\
i
\end{array}\right) \frac{((M-i+1) x-D)^{M}}{M !}
\end{aligned}
$$

We then have (19).

\section{APPENDIX II \\ PROOF OF THEOREM 1}

Proof: From (16), the maximum distortion is only a function of the maximum sample distance $d_{\max }^{(M)}$. Under $\pi_{u}$, the average maximum distortion is given in (18) as

$$
\begin{aligned}
\overline{\mathcal{E}}\left(M, \mathrm{SNR} ; \pi_{u}\right) & =\mathcal{E}\left(\frac{D}{M+1}, \mathrm{SNR}\right) \\
& =\frac{\frac{1}{\mathrm{SNR}}+1-e^{-f D /(M+1)}}{\frac{1}{\mathrm{SNR}}+1+e^{-f D /(M+1)}} \frac{\sigma^{2}}{2 f} .
\end{aligned}
$$

The following expression is used in the later proof.

Fact: Let $g(x)=\left(a-e^{-x}\right) /\left(a+e^{-x}\right)$. Then as $x \rightarrow 0$

$$
g(x)=\frac{a-1}{a+1}+\frac{2 a x}{(a+1)^{2}}+O\left(x^{2}\right) .
$$

The above expression can be shown by applying Taylor's theorem to $g(x)$.

For fixed $f$ and $D$, as $M \rightarrow \infty, f D /(M+1) \rightarrow 0$. By (54), we have

$$
\begin{aligned}
\overline{\mathcal{E}}\left(M, \mathrm{SNR} ; \pi_{u}\right)= & \frac{\sigma^{2}}{2 f} \frac{1}{1+2 \mathrm{SNR}} \\
& +\sigma^{2} \frac{\left(\frac{1}{\mathrm{SNR}}+1\right)}{\left(\frac{1}{\mathrm{SNR}}+2\right)^{2}} \frac{D}{M+1}+o\left(\frac{1}{M}\right) .
\end{aligned}
$$

The excess maximum distortion under $\pi_{u}$ is then given by

$$
\begin{aligned}
\overline{\mathcal{E}}\left(M, \mathrm{SNR} ; \pi_{u}\right) & -\overline{\mathcal{E}}_{\infty}\left(\mathrm{SNR} ; \pi_{u}\right) \\
& =\sigma^{2} \frac{\left(\frac{1}{\mathrm{SNR}}+1\right)}{\left(\frac{1}{\mathrm{SNR}}+2\right)^{2}} \frac{D}{M+1}+o\left(\frac{1}{M}\right) .
\end{aligned}
$$

Under the random sampling $\pi_{r}$, the origins of the $M$ received samples $\left\{P_{1}, \ldots, P_{M}\right\}$ are i.i.d. with uniform distribution $U(0, D)$ and $d_{\max }^{(M)}=\max _{0 \leq i \leq M} d_{i}^{(M)}$. In the following two lemmas, we connect $d_{\max }^{(M)}$ to the maximum of i.i.d. expo- nential random variables and state the almost sure behavior of $d_{\text {max }}^{(M)}$ that is needed to find expression for $r(M, \mathrm{SNR})$.

Lemma 1: Let $\left\{E_{n}\right\}$ be i.i.d. unit exponential random variables and $\Gamma_{n}=E_{1}+\cdots+E_{n}$, for $n \geq 1$. Then, the joint distribution of $\left(\Gamma_{1} / \Gamma_{n+1}, \ldots, \Gamma_{n} / \Gamma_{n+1}\right)$ is the same as that of the order statistics from $n$ i.i.d. random variables with uniform distribution $U(0,1)$, i.e.,

$$
\begin{aligned}
f_{\Gamma_{1} / \Gamma_{n+1}, \ldots, \Gamma_{n} / \Gamma_{n+1}}\left(u_{1}, \ldots, u_{n}\right) \\
= \begin{cases}n !, & \text { if } 0<u_{1}<\cdots<u_{n}<1 \\
0, & \text { otherwise. }\end{cases}
\end{aligned}
$$

Proof: The joint distribution of $E_{1}, \ldots, E_{n}$ is

$$
f_{E_{1}, \ldots, E_{n+1}}\left(x_{1}, \ldots, x_{n+1}\right)=e^{-\sum_{i=1}^{n+1} x_{i}}
$$

for $x_{i}>0,1 \leq i \leq n+1$. Note that $\Gamma_{i}=\sum_{j=1}^{i} E_{j}$. For any $t>0$, let

$$
u_{i}=\frac{1}{t} \sum_{j=1}^{i} x_{j} .
$$

By transformation of random variables, we have the joint density of $\Gamma_{1} / t, \ldots, \Gamma_{n+1} / t$ given by

$$
\begin{array}{r}
f_{\Gamma_{1} / t, \ldots, \Gamma_{n+1} / t}\left(u_{1}, \ldots, u_{n+1}\right) \\
=e^{-t u_{n+1}} t^{n+1}, \quad 0<u_{1}, \ldots<u_{n+1} .
\end{array}
$$

Notice that $\Gamma_{n+1}$ has a gamma density

$$
f_{\Gamma_{n+1}}(t)=\frac{e^{-t} t^{n}}{n !}, \quad t \geq 0
$$

and thus

$$
f_{\Gamma_{n+1} / t}(1)=\frac{e^{-t} t^{n+1}}{n !}, \quad t>0 .
$$

Therefore, from (59) and (60), we have the joint density

$$
\begin{aligned}
f_{\Gamma_{1} / \Gamma_{n+1}, \ldots, \Gamma_{n} / \Gamma_{n+1}} & \left(u_{1}, \ldots, u_{n}\right) \\
& =f_{\Gamma_{1} / t, \ldots, \Gamma_{n} / t \mid \Gamma_{n+1}=t}\left(u_{1}, \ldots, u_{n}\right) \\
& =n !, \quad 0<u_{1}<\cdots<u_{n}<1 .
\end{aligned}
$$

By Lemma 1, if $\left\{P_{1}, \ldots, P_{M}\right\}$ are the origins of the $M$ received samples, then the joint distribution of their order statistics $\left(P_{(1)}, \ldots, P_{(M)}\right)$ is the same as that of $D\left(\Gamma_{1} / \Gamma_{M+1}, \ldots, \Gamma_{M} / \Gamma_{M+1}\right)$. Therefore, the joint distribution of distances $\left(d_{0}^{(M)}, \ldots, d_{M}^{(M)}\right)$, defined in (12), is the same as that of $D\left(\left(\Gamma_{1}-\Gamma_{0}\right) / \Gamma_{M+1}, \ldots,\left(\Gamma_{M+1}-\Gamma_{M}\right) / \Gamma_{M+1}\right)$, where $\Gamma_{0}=0$. Thus, the maximum distance $d_{\max }^{(M)}$ can be represented by

$$
\begin{aligned}
d_{\max }^{(M)} & =\frac{D}{\Gamma_{M+1}} \max _{1 \leq i \leq M+1}\left\{\Gamma_{i}-\Gamma_{i-1}\right\} \\
& =\frac{D}{\Gamma_{M+1}} \max _{1 \leq i \leq M+1} E_{i} .
\end{aligned}
$$

Therefore, we have

$$
(M+1) d_{\max }^{(M)}=D \frac{M+1}{\Gamma_{M+1}} \max _{1 \leq i \leq M+1} E_{i} .
$$


By the strong law of large numbers, we have

$$
\frac{\Gamma_{M+1}}{M+1}=\frac{\sum_{i=1}^{M+1} E_{i}}{M+1} \rightarrow 1 \quad \text { a.s. }
$$

Let $A_{M}=\max _{1 \leq i \leq M} E_{i}$, which is the maximum of i.i.d. exponential random variables. From the asymptotic theory of extreme statistics, we have the almost sure asymptotic property of $A_{M}$ as follows.

Lemma 2 (Logarithmic Growth Rate of $A_{M}$ [18, p. 262], [19, p. 215]: Let $A_{M}$ be the maximum of $M$ i.i.d. unit expo-

$\operatorname{Pr}\left(\liminf _{M \rightarrow \infty}\left[1-\frac{\log \log M}{\log M} \leq \frac{A_{M}}{\log M}<1+\frac{2 \log \log M}{\log M}\right]\right)$

$=1$.

Therefore $^{6}$

$$
\frac{A_{M}}{\log M} \rightarrow 1 \quad \text { a.s. }
$$

Combining (63)-(65), we have

$\operatorname{Pr}\left(\liminf _{M \rightarrow \infty}\left[\left|\frac{(M+1) d_{\max }^{(M)}}{\log M}-D\right|<\frac{2 \log \log M}{\log M}\right]\right)=1$.

It follows that

$$
\frac{(M+1) d_{\max }^{(M)}}{\log M} \rightarrow D \quad \text { a.s.. }
$$

The maximum distortion $\mathcal{E}\left(d_{\max }^{(M)}, \mathrm{SNR}\right)$ in (16) can be rewritten as

$$
\mathcal{E}\left(d_{\max }^{(M)}, \mathrm{SNR}\right)=\frac{\sigma^{2}}{2 f} \frac{\frac{1}{\mathrm{SNR}}+1-e^{-f\left(M d_{\max }^{(M)} / \log M\right)(\log M / M)}}{\frac{1}{\mathrm{SNR}}+1+e^{-f\left(M d_{\max }^{(M)} / \log M\right)(\log M / M)}} .
$$

Therefore, for all large $M$, the average maximum distortion under $\pi_{r}$ is obtained by

$$
\begin{aligned}
& \overline{\mathcal{E}}\left(M, \mathrm{SNR} ; \pi_{r}\right) \\
& =E\left[\mathcal{E}\left(d_{\text {max }}^{(M)}, \mathrm{SNR}\right) \mid M, \pi_{r}\right] \\
& =\frac{\sigma^{2}}{2 f} E\left[\frac{\frac{1}{\mathrm{SNR}}+1-e^{-f\left(M d_{\max }^{(M)} / \log M\right)(\log M / M)}}{\frac{1}{\mathrm{SNR}}+1+e^{-f\left(M d_{\max }^{(M)} / \log M\right)(\log M / M)}}\right] \\
& =\frac{\sigma^{2}}{2 f} \frac{\frac{1}{\mathrm{SNR}}+1-e^{-f(D+O(\log \log M / \log M)) \log M / M}}{\frac{1}{\mathrm{SNR}}+1+e^{-f(D+O(\log \log M / \log M)) \log M / M}}
\end{aligned}
$$

where the last equation is from (67). The limiting distortion $\overline{\mathcal{E}}_{\infty}\left(\mathrm{SNR} ; \pi_{r}\right)$ under $\pi_{r}$ is then given by

$$
\overline{\mathcal{E}}_{\infty}\left(\mathrm{SNR} ; \pi_{r}\right)=\lim _{M \rightarrow \infty} \overline{\mathcal{E}}\left(M, \mathrm{SNR} ; \pi_{r}\right)=\frac{\sigma^{2}}{2 f} \frac{1}{1+2 \mathrm{SNR}} .
$$

${ }^{6} \mathrm{Also}$, from the theory of extreme value distributions, the limiting distribution of normalized $A_{M}$ is

$$
\operatorname{Pr}\left(A_{M}-\log M \leq x\right)=F_{A_{M}}(x+\log M) \rightarrow G_{X}(x)=e^{-e^{-x}} .
$$

Therefore, the excess maximum distortion under $\pi_{r}$ is obtained by

$\overline{\mathcal{E}}\left(M, \mathrm{SNR} ; \pi_{r}\right)-\overline{\mathcal{E}}_{\infty}\left(\mathrm{SNR} ; \pi_{r}\right)$

$=\frac{\sigma^{2}}{2 f} \frac{\frac{1}{\mathrm{SNR}}+1-e^{-f(D+O(\log \log M / \log M)) \log M / M}}{\frac{1}{\mathrm{SNR}}+1+e^{-f(D+O(\log \log M / \log M)) \log M / M}}$

$$
-\frac{\sigma^{2}}{2 f} \frac{1}{1+2 \mathrm{SNR}}
$$

$=\sigma^{2} \frac{\left(\frac{1}{\mathrm{SNR}}+1\right)}{\left(\frac{1}{\mathrm{SNR}}+2\right)^{2}}\left(D+O\left(\frac{\log \log M}{\log M}\right)\right) \frac{\log M}{M}+O\left(\frac{\log ^{2} M}{M^{2}}\right)$

where the last equation is by (54).

Finally, combining (55) and (72), for all large $M, r(M, \mathrm{SNR})$ is obtained by

$$
\begin{aligned}
r(M, \mathrm{SNR})= & \frac{\overline{\mathcal{E}}\left(M, \mathrm{SNR} ; \pi_{r}\right)-\overline{\mathcal{E}}_{\infty}\left(\mathrm{SNR} ; \pi_{r}\right)}{\overline{\mathcal{E}}\left(M, \mathrm{SNR} ; \pi_{u}\right)-\overline{\mathcal{E}}_{\infty}\left(\mathrm{SNR} ; \pi_{u}\right)} \\
= & \left(1+O\left(\frac{\log \log M}{\log M}\right)\right) \log M \\
& +o\left(\frac{\log M}{M^{2}}\right)+O\left(\frac{\log ^{2} M}{M}\right) \\
= & \log M+O(\log \log M)+O\left(\frac{\log ^{2} M}{M}\right) .
\end{aligned}
$$

\section{APPENDIX III \\ PROOF OF COROLLARY 1}

Proof: We set the ratio of excess maximum distortion $r(M, \mathrm{SNR})=1$, i.e.,

$$
\frac{\overline{\mathcal{E}}\left(M_{\pi_{r}}, \mathrm{SNR} ; \pi_{r}\right)-\overline{\mathcal{E}}_{\infty}\left(\mathrm{SNR} ; \pi_{r}\right)}{\overline{\mathcal{E}}\left(M_{\pi_{u}}, \mathrm{SNR} ; \pi_{r}\right)-\overline{\mathcal{E}}_{\infty}\left(\mathrm{SNR} ; \pi_{r}\right)}=1 .
$$

From (72), as $M_{\pi_{r}}$ becomes large, we have

$$
\begin{aligned}
\overline{\mathcal{E}}\left(M_{\pi_{r}}, \mathrm{SNR} ; \pi_{r}\right)-\overline{\mathcal{E}}_{\infty}\left(\mathrm{SNR} ; \pi_{r}\right) \\
=\sigma^{2} \frac{\left(\frac{1}{\mathrm{SNR}}+1\right)}{\left(\frac{1}{\mathrm{SNR}}+2\right)^{2}}\left(D+O\left(\frac{\log \log M_{\pi_{r}}}{\log M_{\pi_{r}}}\right)\right) \frac{\log M_{\pi_{r}}}{M_{\pi_{r}}} \\
\quad+o\left(\frac{\log M_{\pi_{r}}}{M_{\pi_{r}}}\right)
\end{aligned}
$$

and from (56)

$$
\begin{aligned}
\overline{\mathcal{E}}\left(M_{\pi_{u}}, \mathrm{SNR} ; \pi_{u}\right)-\overline{\mathcal{E}}_{\infty}\left(\mathrm{SNR} ; \pi_{u}\right) \\
=\sigma^{2} \frac{\left(\frac{1}{\mathrm{SNR}}+1\right)}{\left(\frac{1}{\mathrm{SNR}}+2\right)^{2}} \frac{D}{M_{\pi_{u}}+1}+o\left(\frac{1}{M_{\pi_{u}}}\right) .
\end{aligned}
$$

Therefore

$$
\frac{\left(D+O\left(\frac{\log \log M_{\pi_{r}}}{\log M_{\pi_{r}}}\right)\right) \frac{\log M_{\pi_{r}}}{M_{\pi_{r}}}+o\left(\frac{\log M_{\pi_{r}}}{M_{\pi_{r}}}\right)}{\frac{D}{M_{\pi_{u}}+1}+o\left(\frac{1}{M_{\pi_{u}}}\right)}=1 .
$$


It follows that

$$
\begin{aligned}
M_{\pi_{u}} & =\frac{M_{\pi_{r}}}{\log M_{\pi_{r}}}\left[\frac{1+o\left(\frac{1}{M_{\pi_{u}}}\right)}{1+O\left(\frac{\log \log M_{\pi_{r}}}{M_{\pi_{r}}}\right)}\right] \\
& =O\left(\frac{M_{\pi_{r}}}{\log M_{\pi_{r}}}\right) .
\end{aligned}
$$

\section{APPENDIX IV \\ PROOF OF COROLLARY 2}

Proof: From Theorem 2, we have $\exists M_{0}$, such that for all $M>M_{0}$

$$
\begin{aligned}
\nu(M, \mathrm{SNR})= & \frac{1}{1+\frac{C_{1}(\mathrm{SNR})}{M+1}}+\frac{C_{1}(\mathrm{SNR})}{1+\frac{C_{1}(\mathrm{SNR})}{M+1}} \frac{\log M}{M} \\
& +O\left(\frac{\log \log M}{M}\right)+O\left(\frac{\log ^{2} M}{M^{2}}\right) \\
= & \frac{1}{1+\frac{C_{1}(\mathrm{SNR})}{M+1}}+\frac{1}{1+\frac{M+1}{C_{1}(\mathrm{SNR})} \log M} \\
& +O\left(\frac{\log \log M}{M}\right)+O\left(\frac{\log ^{2} M}{M^{2}}\right) .
\end{aligned}
$$

If we select $\beta(M)$ such that $M / C_{1}(\beta(M)) \ll 1$, then for SNR $>\beta(M)$, we have (30).

\section{REFERENCES}

[1] L. Tong, Q. Zhao, and S. Adireddy, "Sensor networks with mobile agents," in Proc. 2003 Military Commun. Int. Symp., Boston, MA, Oct. 2003.

[2] Q. Zhao and L. Tong, "Quality-of-service specific information retrieval for densely deployed sensor network," in Proc. 2003 Military Commun. Int. Symp., Boston, MA, Oct. 2003.

[3] P. Venkitasubramaniam, S. Adireddy, and L. Tong, "Sensor networks with mobile agents: Optimal random access and coding," IEEE J. Sel. Areas Commun. (Special Issue on Sensor Networks), vol. 22, no. 6, pp. 1058-1068, Aug. 2004.

[4] E. Masry, "Poisson sampling and spectral estimation of continuoustime processes," IEEE Trans. Inf. Theory, vol. IT-24, pp. 173-183, 1978.

[5] I. Bilinskis and A. Mikelsons, Randomized Signal Processing. Englewood Cliffs, NJ: Prentice-Hall, 1992.

[6] E. Masry, "Ploynomial interpolation and prediction of continuous-time processes from random samples," IEEE Trans. Inf. Theory, vol. 43, pp. 776-783, Mar. 1997.

[7] O. A. Z. Leneman and J. Lewis, "Random sampling of random processes: Mean-square comparison of various interpolators," IEEE Trans. Autom. Control, vol. AC-11, pp. 396-403, 1966.

[8] O. A. Z. Leneman, "Random sampling of random processes: Optimum linear interpolation," J. Frank. Inst., vol. 281, pp. 302-314, 1966.

[9] M. Micheli and M. I. Jordan, "Random sampling of a continuous-time stochastic dynamical system," in Proc. 15th Int. Symp. Math. Theory Networks Syst. (MTNS 2002), South Bend, IN, Aug. 2002.

[10] A. V. Balakrishnan, "A note on the sampling principle for continuous signals," IEEE Trans. Inf. Theory, vol. IT-3, pp. 143-146, 1957.

[11] B. Liu and T. Stanley, "Error bounds for jittered sampling," IEEE Trans. Autom. Control, vol. AC-10, pp. 449-454, 1965.

[12] A. Woo and D. Culler, "A transmission control scheme for medium access in sensor networks," in Proc. ACM/IEEE Conf. Mobile Commun. Network., Rome, Italy, Jul. 2001, pp. 221-235.

[13] W. Ye, J. Heidemann, and D. Estrin, "An energy-efficient MAC protocols for wireless sensor networks," in Proc. IEEE INFOCOM, New York, Jun. 2002, pp. 1567-1576.

[14] K. Sohrabi, J. Gao, V. Ailawadhi, and G. Pottie, "Protocols for selforganization of a wireless sensor network," IEEE Personal Commun., pp. 16-27, Oct. 2000.
[15] R. Iyer and L. Kleinrock, "QoS control for sensor networks," in Proc. 2003 ICC, May 2003.

[16] D. Cox and H. Miller, The Theory of Stochastic Processes. New York: Wiley, 1965.

[17] S. Reshick, Advenures in Stochastic Processes. Boston, MA: Birkhäuser, 1994.

[18] J. Galambos, The Asymptotic Theory of Extreme Order Statistics, 2nd ed. Melbourne, FL: Krieger, 1987.

[19] S. Resnick, A Probability Path. Boston, MA: Birkhäuser, 2001.

[20] P. Billingsley, Probability and Measure. New York: Wiley Inter-Science, 1995, vol. 3.

[21] M. Dong, L. Tong, and B. M. Sadler, "Information retrieval and processing in sensor networks: Deterministic scheduling vs. random access," in IEEE Int. Symp. Inf. Theory (ISIT), 2004.

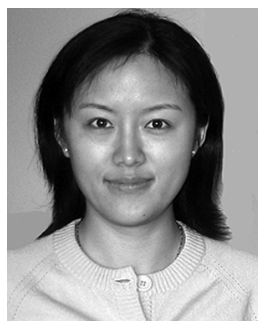

Min Dong (S'00-M'05) received the B.Eng. degree from Tsinghua University, Beijing, China, in 1998 and the $\mathrm{Ph} . \mathrm{D}$. degree in electrical and computer engineering from Cornell University, Ithaca, NY, in 2004.

She is currently with Corporate Research and Development, Qualcomm, Inc., San Diego, CA, where she has been primarily involved in the orthogonal-frequency-division multiple-access (OFDMA) system design and performance analysis and has coinvented several pending patents. Her research interests are in the areas of signal processing, wireless communications, and mobile networks.

Dr. Dong received the 2004 IEEE Signal Processing Society Best Paper Award.

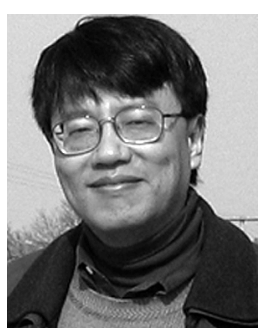

Lang Tong (S'87-M'91-SM'01-F'05) received the B.E. degree from Tsinghua University, Beijing, China, in 1985 and the M.S. and Ph.D. degrees in electrical engineering from the University of Notre Dame, Notre Dame, IN, in 1987 and 1991, respectively.

$\mathrm{He}$ is a Professor in the School of Electrical and Computer Engineering, Cornell University, Ithaca, NY. He was a Postdoctoral Research Affiliate with the Information Systems Laboratory, Stanford University, in 1991. He was the 2001 Cor Wit Visiting Professor at the Delft University of Technology, The Netherlands. His areas of interest include statistical signal processing, wireless communications, communication networks and sensor networks, and information theory.

Dr. Tong received the Young Investigator Award from the Office of Naval Research in 1996, the Outstanding Young Author Award from the IEEE Circuits and Systems Society in 1991, the 2004 IEEE Signal Processing Society Best Paper Award (with M. Dong), the 2004 Leonard G. Abraham Prize Paper Award from the IEEE Communications Society (with P. Venkitasubramaniam and S. Adireddy). He is an Associate Editor of the IEEE TRANSACTIONS ON Signal PROCESSING and IEEE SignAL PROCESSING LETTERS.

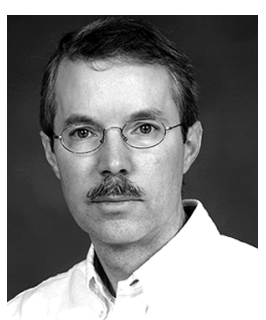

Brian M. Sadler (M'90-SM'02) received the B.S. and M.S. degrees from the University of Maryland, College Park, and the Ph.D. degree from the University of Virginia, Charlottesville, all in electrical engineering.

$\mathrm{He}$ is a Senior Research Scientist with the Army Research Laboratory, Adelphi, MD, and lectures at The Johns Hopkins University, Baltimore, MD. His research interests include signal processing for mobile wireless and ultra-wide-band systems and sensor signal processing and networking.

$\mathrm{He}$ is an Associate Editor of IEEE Signal Processing LetTERs and was an Associate Editor of the IEEE TRANSACTIONS ON SignAl PROCEsSING. He has been a Guest Editor for the IEEE JouRnal ON SELECTED AREAS IN COMMUNICATIONS and the IEEE Signal Processing Magazine and is a Member of the IEEE Signal Processing Society Sensor Array and Multi-Channel Technical Committee. He is a member of the Editorial Board of the EURASIP Journal on Wireless Communications and Networking and the International Journal of Sensor Networks. 